\title{
Dissecting the Reduced Ovarian Reserve Seen with Ovarian Enometriomas with That Caused by Surgical Excision of Ovarian Enometriomas- What Comes First
}

\author{
Kulvinder Kochar Kaur ${ }^{1 *}$, Gautam Allahbadia ${ }^{1}$ and Mandeep Singh ${ }^{2}$ \\ ${ }^{1}$ Scientific Director, India \\ ${ }^{2}$ Consultant Neurologist, India \\ *Corresponding author: Kulvinder Kochar Kaur, Scientific Director, India
}

Submission: December 26, 2018; Published: January 09, 2019

\section{Opinion}

There have been concerns raised regarding the possibility of surgical excision of the endometrioma causing damage to the ovarian reserve. Three recent systematic reviews reported a decrease in serum anti mullerian hormone (AMH) levels as compared to preoperative levels after endometrioma excision [13]. But very recently Muzli et al. [4], gave a meta-analysis on the peripheral levels of AMH in women having ovarian endometriomas. In contrast to earlier met-analyses they reported lower levels of $\mathrm{AMH}$ as compared to controls and in women having any other ovarian cysts which suggests that what is reported following surgery, the damage to the ovarian reserve ,may at least in part be present before any intervention. Although these results might answer a lot of unresolved questions, they need to be interpreted using some caution.

This study by Muzli et al. [4] shows that there is a lower serum AMH in women having endometriomas. But they do not show that ovarian reserve is injured in women having endometriomas. Thus, one might hypothesize that the presence of these endometriomas may not directly damage the ovarian reserve but might disturb the physiology of the ovary which =>temporary derangement of the complex and yet unknown mechanisms that regulate AMH production. Further one needs to consider the importance of changes in local vascularization that is induced by large endometriomas. It may be that AMH is normally produced in the ovaries carrying these endometriomas but may have difficulties in reaching the peripheral circulation. Since AMH is a paracrine factor and not a typical hormone, hence if there are any impaired secretion processes of AMH they do not get compensated by feedback mechanisms, like other hormones. Hence till date there is not enough evidence to say that the decreased serum $\mathrm{AMH}$ in women carrying endometriomas actually portray any definitive damage to the ovarian reserve but only a temporary and potentially reversible interruption of ovarian physiology. As highlighted by
Muzli et al. [4] role of timing is important i.e. the comparison of age of endometriomas on the effect on levels of serum AMH, although they could not resolve this problem. Clinically doing this will support causality, but unfortunately the possible role of time related damage induced by endometriomas has not got much attention and when done occasionally contrasting data has been published by Benaglia et al. [5] \& Kasapoglu et al. [6] in a very recent study [5,6]. Another important aspect of this study by Muezli et al. [4] is the size of endometriomas considered. In their meta-analysis the mean diameter of endometriomas considered is large [which exceeds $5 \mathrm{~cm}$ in the majority]. As said by Muzli et al. [4] this has not got enough attention earlier, but might play a significant role. It is important to understand that how volumes vary with the increasing size of endometriomas, e.g. once size is $6 \mathrm{~cm}$ then volume of endometriomas is 27 -fold greater to that in comparison of volume of endometriomas of $2 \mathrm{~cm}$ (about $113 \mathrm{ml}$ and $4 \mathrm{ml}$ respectively). Also, importantly these cysts develop within an organ namely ovary whose basal volume is $4 \mathrm{ml}$. Hence this might explain the differences seen in metanalyses by Muzli [4] and Humaidan in previous evidence obtained in in vitro fertilization showing minimum impact by ovarian endometriomas. Most studies focused on ovarian endometriomas of $2 \mathrm{~cm}$ in IVF studies. In a rare study where large ovarian endometriomas $>5 \mathrm{~cm}$ were considered, a decreased responsiveness was seen in the affected ovaries by Ferrero et al. [7].

Still there are lacunae in Muzli et al. [4]. There are methodological errors which doesn't allow one to believe the estimation of magnitude of this reduction of AMH seen with ovarian endometriomas. High heterogeneity of the meta-analysis used, use of parametric statistics as serum AMH gets positively skewed, along with unilaterality of the lesion are not all helpful in being able to reliably conclude that this effect on AMH is being caused by ovarian endometriomas per sec. One needs to disentangle the effect ovarian endometriomas vis a vis that of surgery on reduced ovarian reserve. 


\section{Conclusion}

In conclusion although this study by Muzli et al. [4] tries to move further by showing that probably ovarian endometriomas might be the culprits in reducing ovarian reserve directly, but one should not jump to the conclusions that surgery per se does not have a detrimental effect. There is a need to keep working on to dissect the roles of each and the relationship of ovarian endometriomas, surgery and ovarian reserve. Further the role of time on deterioration of $\mathrm{AMH}$ with coexisting ovarian endometriomas and whether the effect of reduced ovarian reserve is permanent or temporary with either ovarian endometriomas itself or surgery per se and other factors associated with surgery with ovarian endometriomas like involvement of mesosalpinx in this reduction [8], and how one can prevent the decreased in ovarian reserve either by conservative methods or preventing surgery induced reduction in ovarian reserve.

\section{References}

1. Raffi F, Metwally M, Amer S (2012) The impact of excision of ovarian endometrioma on ovarian reserve: A systematic review and metaanalyses. J Clin Endocrol Metab 97(9): 3146-3154.

2. Somigliana E, Berlanda N, Benaglia L, Vigano P, Vercellani P, et al. (2012) Surgical excision of endometriomas and ovarian reserve. A systematic review on serum anti mullerian hormone level modifications. Fertil Steril 98(6): 1531-1538.
3. Hamdan M, Dunselman G, Li TC, Cheng Y (2015) The impact of endometrioma on IVF/ICSI outcomes: A systematic review and metaanalyses. Hum Reprod Update 21(6): 809-825.

4. Muzli L, Di Tucci C, Di Feliciantonio M, Galali G, Di Donato V, et al. (2018) Antimullerian hormone is reduced in the presence of ovarian endometriomas: A systematic review and meta-analyses. Fertil Steril 110(5): 932-940.

5. Benaglia L, Castiglioni M, Paffoni A, Sarais V, Vercellini P, et al. (2017) Is endometrioma-associated damage to ovarian reserve progressive? Insights from IVF cycles. Eur J Obstet Gynecol Reprod Biol 217: 101-105.

6. Kasapoglu I, Ata B, Uyanklar O, Seyhan A, Orhan A, et al. (2018) Endometrioma-related reduction in ovarian reserve (ERROR): A progressive longitudinal study. Fertil Steril 110(1): 122-127.

7. Ferrrero S, Scala C, Tafi E, Racca A, Venturini PL, et al. (2017) Impact of large ovarian endometriomas on the response to superovulation for in vitro fertilization: A retrospective study. Eur J Obstet Gynecol Reprod Biol 213: 17-21.

8. Saito A, Iwase A, Nakamura T, Osuka S, Murase T, et al. (2016) Involvement of mesosalpinx in endometrioma is a possible risk factor for decrease of ovarian reserve after cystectomy: A retrospective cohort study. Reprod Biol and Endocrinol 14: 72
Creative Commons Attribution 4.0 International License

For possible submissions Click Here

\section{Submit Article}

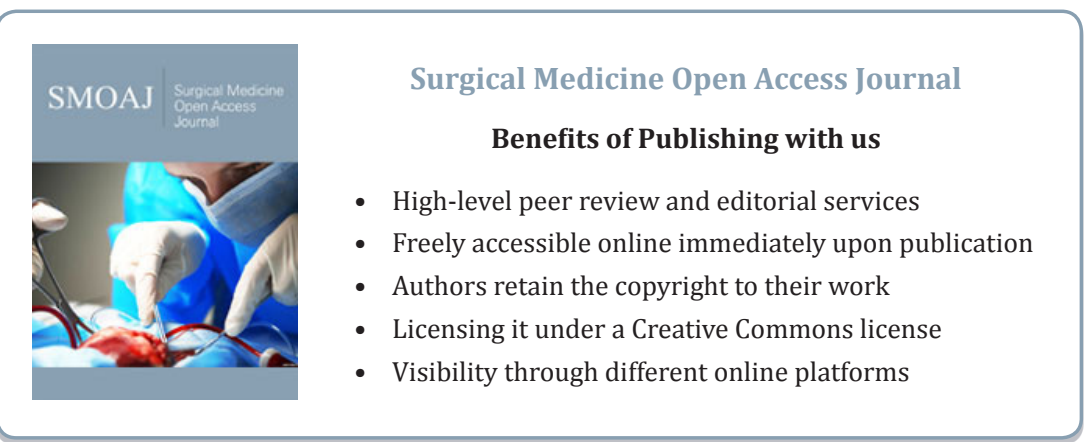

